\title{
Qualitative research methods
}

David F Marks

Potential competing interests: The author(s) declared that no potential competing interests exist.

Qualitative research methods aim to understand the meanings, purposes and intentions of behaviour, not its amount or quantity. A huge variety of methods are available: diaries and blogs; discourse analysis; focus groups; grounded theory; historical analysis; interpretative phenomenological analysis; interviews, especially semi-structured; and narrative approaches. 